\title{
Rechtsgeschichte
}

\section{Barbara Frenz}

\section{Absage an die »Herrengewalt«}


sendjährigen Kampf zweier Prinzipien, zum Kampf zwischen freiem Wettbewerb und Wettbewerbsvermeidung, in dem sich dann schließlich doch das Gute durchsetzt - ein Grundmuster des Erzählens, das wahrlich zu den archaischen Anfängen historischer Reflexion zurückführt.

Thomas Simon

\section{Absage an die »Herrengewalt «*}

Seit der Studie Otto Brunners ${ }^{\mathrm{I}}$ ist der Fehde führende Adlige rechtshistorisch rehabilitiert. Anders steht es um das Bild des seiner Herrschaft unterworfenen Bauern: Ihm als dem unter grundherrlichem »Schutz und Schirm « Stehenden war - wie auch dem Bürger - das »subsidiäre Rechtsmittel « angeblich nur in der Ausnahmeform von Blutrache und Totschlagsfehde erlaubt. ${ }^{2}$ Der »neutralere « Kontext einer regulären Interessenkollision zwischen Parteien, den die Konfliktforschung nahe legt, wird für bäuerliches Handeln nicht in Erwägung gezogen; einen aktiven Part bei der Durchsetzung eigener Ansprüche erkennt man dabei höchstens im kollektiven »Widerstand «. Eindimensionale soziale Rollenzuschreibungen bestimmen den Forschungshorizont. Nie ist z. B. die Frage gestellt worden, ob ein mittelalterlicher oder frühneuzeitlicher Mensch tatsächlich der Waffenfähigkeit oder Waffen bedurfte, um eine Fehde zu führen. So weckt der "gemeine Mann « in der viel besprochenen Untersuchung von Gadi Algazi $^{3}$ zwar erstmals überhaupt das nähere Interesse einer Untersuchung zum Fehdewesen, tritt jedoch auch da nur als Objekt einer sich sozial reproduzierenden »Herrengewalt « in Erscheinung. Algazis Ansatz hat allerdings Denkanstöße gegeben, ohne welche wohl gerade Christine Reinles Habilitationsschrift nicht jene konzeptionelle Konturschärfe gewonnen hätte, die sie kennzeichnet. Selbstredend setzt sich die Autorin darin nicht nur mit Algazi auseinander - auch wenn dieser mit Abstand ihr Hauptgegner bleibt. Die zugegeben nicht sehr quellengesättigte These des Historikers steht auf einer Beobachtungslinie mit den Studien von Joseph Morsel und Hillay Zmora. ${ }^{4}$ Allen dreien geht es, grob gesagt, um die soziale statt die seit Brunner oft strapazierte rechtliche Funktion der Fehden - um die Beobachtung einer engen Verknüpfung dieser Konfliktpraxis mit sozialer Stratifikation und adliger Herrschaftsbildung.

Gleich zu Beginn ihrer Studie legt Christine Reinle dar, dass sie dieser »funktionsgeschichtliche« Weg nicht überzeugt (2I). Ihre »Bezugsgrößen des Fehdewesens " heißen Recht und soziale Norm ( 56 ff.). Den Ausgangspunkt bilden ungedruckte Quellen - vornehmlich aus dem Niederbayern der Mitte des I5. bis ins erste Jahrzehnt des I6. Jahrhunderts, die im Anhang der Arbeit auf I70 Seiten tabellarisch dokumentiert sind: Bayern-spezifische Gerichtsrechnungen, ausgestellt von den Landschreibern der Rentmeisterämter Burghausen, Ingolstadt, Landshut und Wasserburg, die hierin ihre Einnahmen aus den so genannten Vitztumhändeln verzeichneten. Bei den Einnahmen handelt es sich um in Geldbußen arbiträr umgewandelte Strafen (wandel), die nichtadligen Personen drohten, wenn diese auf außergerichtlichem
* Christine Reinle, Bauernfehden. Studien zur Fehdeführung Nichtadliger im spätmittelalterlichen römisch-deutschen Reich, besonders in den bayerischen Herzogtümern (VSWG-Beihefte I70), Wiesbaden: Steiner 2003, 589 S., ISBN 3-5 I 5-07840-I

I OtTo Brunner, Land und Herrschaft, 5. Aufl. Darmstadt I 984.

2 BrunNer (Fn. I) 62.
3 Gadi Algazi, Herrengewalt und Gewalt der Herren im späten Mittelalter. Herrschaft, Gegenseitigkeit und Sprachgebrauch (Historische Studien, Bd. I7), Frankfurt, New York 1996.

4 Joseph Morsel, "Das sy sich mitt der besstenn gewarsamig schicken, das sy durch die widerwertigenn Franckenn nitt nidergeworffen werdenn. « Überlegungen zum sozialen Sinn der Fehdepraxis am
Beispiel des spätmittelalterlichen Franken, in: Strukturen der Gesellschaft im Mittelalter, hg. von DiETER RöDEL und JOACHIM SCHNEIDER, Wiesbaden I996, I40-I67; Hillay Zmora, State and Nobility in Early Modern Germany: the Knightly Feud in Franconia I440-I 567, Cambridge 1997 . 
Weg und gewaltsam ihre Ansprüche durchzusetzen oder ihre Ehre wiederherzustellen versuchten (70 ff., 23 I ff.). In den Rechnungen fand Christine Reinle zahlreiche Fehden sowie, z. B. aufgrund fehlender Absage, als »fehdeanalog " bezeichnete Handlungen. Und sofern es sich nicht um Bürger handelt, lassen die Akteure die Bezeichnung "Bauer « zumindest nicht abwegig erscheinen. Reinle konstatiert, dass die Landschreiberrechnungen eine genaue soziale Zuordnung nicht ermöglichen $(294 / 5)$. Auch urkundliches Material, zum Teil aus den angrenzenden Gebieten Bayerns, zählt zur induktiven Basis der Untersuchung: vor allem Hafturfehdebriefe, zudem einige Fehdebriefe und Urgichten. Die soziale Identifizierung geschah hier nach dem Ausschließungsprinzip `kein Adliger`, ’kein eventueller Niederadliger`, ’keiner aus dem Söldnermilieu< (362). Anhand eines klar definierten Rasters sammelte die Autorin aus ihren Quellen spezifische für »Fehde « und »fehdeanaloges Handeln « in Frage kommende Verhaltensweisen, wie z. B. das Austreten, das Absagen u.a.m. (245 ff., 359 ff.). Die Auswertung des aufbereiteten Materials nimmt den zweiten und zugleich instruktiven Teil der "phänomenologischen « ( 44 f. u.ö.) Untersuchung ein. Den ersten Teil bilden »Mikrostudien « - Darstellungen von außergewöhnlich gut überlieferten Fällen »irregulärer « bzw. »nichtadliger « Fehden (I 23) und von »Grenzfällen «, die irgendwo zwischen nichtadliger Fehde und Kriminalität angesiedelt sind (202 ff.). Die geographisch breit gestreuten Fallbeispiele verstehen sich als narrative Ergänzung dessen, worüber die Masse der - längst nicht durchweg spröden! - seriellen Quellen schweigt. Wenig überzeugend ist die Ebene der »nichtadligen« bzw. »irregulären« Fehde, auf der hier operiert wird, um das Phänomen »Bauernfehde « noch differenzierter und konturschärfer heraus- zuarbeiten. So stellt auch Reinle fest, dass die Fehden Bürgerlicher ein »lohnenswertes Objekt der Forschung « bilden (357). Mit den Bauernfehden können diese also nicht einfach gleichgeschaltet werden. Die Autorin hätte sich auf ihren eigentlichen Untersuchungsgegenstand beschränken und z.B. die ohnehin oft traktierte Kohlhase-Fehde weglassen sollen. Mit quälenden 74 Seiten unnötig raumgreifend ist auch das einleitende erste Kapitel, das z. B. ausführlich auf den Arbeitsbegriff des "Brauchs " einstimmt (49 ff.), der jedoch in der Untersuchung eher untergeht.

Gleichwohl haben die »Bauernfehden « entscheidend Neues zu bieten. Zum Beispiel die Beobachtung, dass »bäuerlicher Waffenbesitz im Spätmittelalter in einem Umfang gegeben war, der die Möglichkeit zur Fehdeführung zuließ« (36). Und vor allem gibt das von Reinle herangezogene, die »Bauern « betreffende Material detaillierte Einblicke in die Konfliktpraxis dieser sozialen Gruppe. Es handelt es sich dabei ausschließlich um solche Konflikte, deren Austrag obrigkeitlich abgestraft wurde (324). Die unerkannten Bauernfehden sind also noch hinzuzudenken, allerdings unter Berücksichtigung der Tatsache, dass in den bayerischen Herzogtümern der Untersuchungszeit die dörfliche Präsenz der obrigkeitlichen Beamten »dominant « war (304). Von einem regelmäßigen Erfolg der Fehden ist dennoch auszugehen, bedenkt man die Vielzahl ihrer überlieferten Fälle (324).

Als typisches ritualisiertes Element der Fehden (oder »fehdeanalogen Handlungen «) ist das Austreten aus dem dörflichen Rechtsverband bei einer beeindruckend hohen räumlichen Mobilität und unter Ausnutzung eines beträchtlichen sozialen Netzes von Unterstützern, zu denen auch Adlige zählen konnten, hervorzuheben. Des Weiteren die offene Absage durch Anbrin- 
gen von Brandsymbolen oder Fehdebriefen und - wenn es denn hierzu noch kam - die Schädigung des Gegners durch Brandstiftung.

Bemerkenswert ist, dass die zahlreichen Bauernfehden meistens ohne Bewaffnung geführt wurden und primär auf »das wirtschaftliche Substrat des Gegners" abzielten (6I). Es bedarf also nicht mehr unbedingt »des Blickes auf die Blutrachefehde (...), um das Problem bäuerlichen Fehderechts zu erörtern« (350). Überwiegend ging es dabei um strittige, die Subsistenz sichernde Rechtsansprüche (spruch, 278 f.). Und zumindest die »Mikrostudien « zeigen, dass man im Vorfeld für gewöhnlich »geredet, gestritten und auch prozessiert « (286) hat. Unbeantwortet bleibt allerdings die Frage, warum eigentlich im bäuerlichen Milieu der gerichtliche Weg so oft nicht (zu Ende) gegangen wurde.

Häufig nachweisbar sind zwischenbäuerliche Fehden, jedoch auch solche zwischen Bauern und (insbesondere klösterlichen) Herren. Gerne hätte man mehr über den Zusammenhang der Fehde als einem von Bauern gewählten Weg des individuellen Konfliktaustrags mit dem sozialen Status des Konfliktgegners erfahren. Das Problem der sozialen Herkunft der Fehdegegner handelt Reinle jedoch leider nur beiläufig $a b$ (3 I I ff.).

Dagegen gelingt es ihr deutlich zu machen, dass die eigentliche Gegenseite der bäuerlichen Fehdepraxis eine zunehmend härter durchgrei- fende Gesetzgebung ( 75 ff.) und Gerichtspraxis war - insbesondere gegenüber Fremden als potentiellen Fehdern (3 I9). Die Bauern gingen mit ihrem Weg der Selbsthilfe ein immer höheres Risiko des sozialen Abstiegs ein, da ihnen die Rückkehr in die dörfliche Rechtsgemeinschaft von obrigkeitlicher Seite nun zunehmend abgeschnitten wurde. So werden im I6. Jahrhundert die Fälle seltener, in denen sich Fehder in die herzogliche Landshuld zurückkaufen konnten (336f.) - und damit auch der obrigkeitliche Kompromiss zwischen den eigenen Normen und denen der Untertanen (345). Zugleich mehren sich solche Fehden, die eine ungewöhnlich lange Dauer von über zwei Jahren kennzeichnet und die hiermit nahtlos in die berufliche Kriminalität übergingen. Konsequenzen, wie die Autorin lakonisch schlussfolgert, welche die Landesherren beim Ausbau ihrer Herrschaft und der Implementierung ihrer Normen billigend in Kauf nahmen (358).

Die strikte Konzentration auf das zweigleisige Normensystem, von dem aus Christine Reinle das zweckrationale Institut der bäuerlichen Fehde mitsamt seinem frühneuzeitlichen Wandel offen legt, provoziert die Frage nach dem sozialen Sinn dieser Konfliktpraxis. Das aber ist jene historisierende Denkrichtung, deren systematischer Verfolgung sich die Autorin verschlossen hat.

Barbara Frenz 